\title{
Correction to: Generic family with robustly infinitely many sinks
}

\author{
Pierre Berger $^{1}$
}

Received: 14 September 2016 / Accepted: 22 July 2019 / Published online: 15 August 2019

(C) Springer-Verlag GmbH Germany, part of Springer Nature 2019

\section{Correction to: Invent. Math.} https://doi.org/10.1007/s00222-015-0632-6

All the main results of [2] are correct, but this would need a substantial variation of the proof as done in [3]. In this corrigendum, we prefer to change the topologies considered in all the statements of [2]. Also we generalize and correct the fundamental property of parablender.

Correction of the statements For $d, r, k \geq 0$, and $M, N$ Riemaniann manifolds, two different spaces of $C^{d}$-families $\left(f_{a}\right)_{a}$ of $C^{r}$-maps $f_{a} \in C^{r}(M, N)$ parametred by $\mathbb{R}^{k}$, can be defined as follows:

$$
\begin{gathered}
C_{A}^{d, r}\left(\mathbb{R}^{k}, M, N\right)=\left\{\left(f_{a}\right)_{a}: \partial_{a}^{i} \partial_{z}^{j} f_{a}(z)\right. \text { exists continuously for all } \\
\left.i \leq d, i+j \leq r \text { and }(a, z) \in \mathbb{R}^{k} \times M\right\} \\
C_{P S}^{d, r}\left(\mathbb{R}^{k}, M, N\right)=\left\{\left(f_{a}\right)_{a}: \partial_{a}^{i} \partial_{z}^{j} f_{a}(z)\right. \text { exists continuously for all } \\
\left.j \leq r, i \leq d \text { and }(a, z) \in \mathbb{R}^{k} \times M\right\}
\end{gathered}
$$

We endow these spaces with the compact open topology w.r.t. the considered derivatives.

The original article can be found online at https://doi.org/10.1007/s00222-015-0632-6.

$\triangle$ Pierre Berger

pierre.berger@normalesup.org

1 Université Paris 13, 93430 Villetaneuse, France 
We notice that in the important case $r=\infty$ these two spaces are equal. In general, it holds:

$$
C_{A}^{d, d+r} \subset C_{P S}^{d, r} \subset C_{A}^{d, r}
$$

The previous article dealt with the space $C_{P S}^{d, r}$; the space $C_{A}^{d, r}$ should be considered instead of $C_{P S}^{d, r}$. Then the whole article is correct, but the statement of the fundamental property of the parablender which needs $1 \leq d<r \leq \infty$ (the case $d=r$ does not seem to work).

Therefore, the topology involved in the statement of Theorems A, C (and facts 4.2, 4.3, 4.4 of its proof) must be corrected to $C_{A}^{d, r}$ for any $1 \leq d<r \leq$ $\infty$. Likewise, the topology involved in the statement of Theorems B must be corrected to $C_{A}^{d, r}$ for any $1 \leq d<r<\infty$.

This correction removes the case $d=r \geq 2$. However, the variation [3] of [2] gives the case $d=r \geq 1$ and also $d \geq r$ for the topologies $C_{A}^{d, r}$ and $C_{P S}^{d, r}$. In particular the statements of the main theorems of the article under correction are correct.

Here is the mistake I made. The space $C_{P S}^{d, r}$ is actually not stable by composition. For instance, if $N=M$, there exists $\left(f_{a}\right)_{a} \in C_{P S}^{d, r}$ such that $\left(f_{a} \circ f_{a}\right)_{a}$ does not belong to $C_{P S}^{d, r}$. That is why we correct it by the space $C_{A}^{d, r}$ which is stable by composition.

I am grateful to S. Crovisier for valuable suggestions on the presentation of the following section.

\section{Correction and generalization of the fundamental property}

Let us fix $k \geq 0,0 \leq d<r \leq \infty$. Given a Riemannian manifold $M$, and $C^{d}$-families of points $\left(z_{a}\right)_{a \in \mathbb{R}^{k}}$, its $C^{d}$-jet at $a=0$ is denoted by $J_{0}^{d}\left(z_{a}\right)_{a}=$ $\sum_{j=0}^{d} \frac{\partial_{a}^{j} z_{a}}{j !} a^{\otimes j}$. Let $J_{0}^{d} M$ be the space of $C^{d}$-jets of $C^{d}$-families of points in $M$ at $a=0$.

We notice that any $C_{A}^{d, r}$-family $\left(f_{a}\right)_{a}$ of $C^{r}$-maps $f_{a}$ of $M$ acts canonically $J_{0}^{d} M$ as the map:

$$
J_{0}^{d}\left(f_{a}\right)_{a}: J_{0}^{d}\left(z_{a}\right)_{a} \in J_{0}^{d} M \mapsto J_{0}^{d}\left(f_{a}\left(z_{a}\right)\right)_{a} \in J_{0}^{d} M
$$

Let us define a category of $C^{d}$-parablenders containing those of [1,2]. To this end, put $I_{e}=[-1,1], Y_{e}=I_{e} \times I_{e}, \partial^{s} Y_{e}=\partial I_{e} \times I_{e}$ and $\partial^{u} Y_{e}=I_{e} \times \partial I_{e}$. 
Definition 1 An affine $C^{d}$-para-IFS of $\mathbb{R}^{2}$ is a finite set of family of maps $\left\{\left(\stackrel{\circ}{g}_{a}^{\delta}\right)_{a \in \mathbb{R}^{k}}: \delta \in \Delta\right\}$ from planar subsets $Y^{\delta}(a)$ onto $Y_{\delta}(a)$ of the form:

$\stackrel{\circ}{g}_{a}^{\delta}:(x, y) \in Y^{\delta}(a)=I_{e} \times I^{\delta}(a) \mapsto\left(\Lambda_{\delta}(a) \cdot x+p_{\delta}(a), \frac{y-q_{\delta}(a)}{\lambda_{\delta}(a)}\right) \in Y_{\delta}(a)=I_{\delta}(a) \times I_{e}$, where $I_{\delta}:=\left[p_{\delta}-\Lambda_{\delta}, p_{\delta}+\Lambda_{\delta}\right], I^{\delta}:=\left[q_{\delta}-\lambda_{\delta}, q_{\delta}+\lambda_{\delta}\right]$, the functions $\lambda_{\delta}, \Lambda_{\delta}, p_{\delta}, q_{\delta}$ are polynomial such that $\left|\Lambda_{\delta}(0)\right|<1 / 2<\left|\lambda_{\delta}(0)\right|<1$ and such that, with $\partial^{u} Y_{\delta}:=I_{\delta} \times \partial I_{e}$ and $\partial^{s} Y^{\delta}:=\partial I_{e} \times I^{\delta}$, there exist compact neighborhoods $A^{\prime} \Subset A \subset J_{0}^{d} \mathbb{R}^{2}$ of 0 satisfying:

(i) for every $J_{0}^{d}\left(z_{a}\right)_{a} \in A$, there exists $\delta \in \Delta$ such that $J_{0}^{d}\left(\stackrel{g}{g}_{a}^{\delta}\left(z_{a}\right)\right)_{a}$ is in $A^{\prime}$.

(ii) For every $\delta \in \Delta$, for every $a$ small, the subsets $Y_{\delta}(a)$ and $Y^{\delta}(a)$ are included in $Y_{e}$, with $\partial^{u} Y_{\delta}(a) \Subset \partial^{u} Y_{e}$ and $\partial^{s} Y^{\delta}(a) \Subset \partial^{s} Y_{e}$.

(ii) for every $Z=\sum_{i=0}^{d} z_{i} a^{\otimes i} \in A$, the value $z_{0}$ belongs to the interior of $Y^{\delta}(0)$ for every $\delta \in \Delta$.

Definition 2 Let $r \geq d$. A family $\left(f_{a}\right)_{a}$ of local diffeomorphisms of $\mathbb{R}^{2}$ defines an affine-like $C^{d}$-parablender if a finite set of its inverse branches $\left\{\left(g_{a}^{\delta}\right)_{a}: \delta \in\right.$ $\Delta\}$ is a $C_{A}^{d, r}$-perturbation of an affine $C^{d}$-para-IFS $\left\{\left(g_{a}^{\circ}\right)_{a}: \delta \in \Delta\right\}$.

Then, for small $a \in \mathbb{R}^{k}$ and $\delta \in \Delta$, with $\hat{I}^{\delta}$ a small neighborhood of $I^{\delta}$, the image by $g_{a}^{\delta}$ of $[-1,1] \times \hat{I}^{\delta}$ intersects $Y_{e}$ at a set $Y_{\delta}\left(f_{a}\right)$ close to $Y_{\delta}(a)$. The set $Y_{\delta}\left(f_{a}\right)$ is bounded by two segments $\partial^{u} Y_{\delta}\left(f_{a}\right)$ of $\partial^{u} Y_{e}$, and two curves $\partial^{s} Y_{\delta}\left(f_{a}\right)$ close to $\partial^{s} Y_{\delta}(a)$. The image by $f_{a}$ of $Y_{\delta}\left(f_{a}\right)$ is denoted by $Y^{\delta}\left(f_{a}\right)$. It is a filled square close to $Y^{\delta}(a)$. The set $Y^{\delta}\left(f_{a}\right)$ is bounded by two segments $\partial^{s} Y^{\delta}\left(f_{a}\right)$ of $\partial^{s} Y_{e}$ and two segments $\partial^{u} Y^{\delta}\left(f_{a}\right)$ close to $\partial^{u} Y^{\delta}(a)$.

We notice that (i)-(ii)-(iii) are still satisfied by $\left(g_{a}^{\delta}\right)_{a}$ instead of $\left(g_{a}^{\delta}\right)_{a}$ and $Y_{\delta}\left(f_{a}\right)$ instead of $Y_{\delta}(a)$.

Then, for every $\underline{\delta}=\left(\delta_{i}\right)_{i \leq-1} \in \Delta^{\mathbb{Z}^{-}}$, we define the following local unstable manifold:

$$
W_{l o c}^{u}\left(\underline{\delta} ; f_{a}\right):=\bigcap_{n \geq 1} f_{a}^{n}\left(Y_{\delta_{-n}}\left(f_{a}\right)\right) .
$$

Example 3 In [1], we showed an example of affine-like $C^{d}$-parablender with Card $\Delta=2$. It is precisely for this example that we consider the topology on the inverse branches rather than on the dynamics, since the degenerate case $\Lambda_{\delta}=0$ does occur in the limit of a renormalization process.

Example 4 In [2], we defined in $\$ 2.2$, the family of maps $\left(f_{a \epsilon}\right)_{a}$ for $f \in U_{0}$ and $\epsilon>$ small enough. The covering property $(i)$ is shown in section $\$ 2.3 .2$ $A=\left\{P \in J_{0}^{2} \mathbb{R}^{2}: P(0) \in[-1,1] \times[-2 / 3,2 / 3] \quad \partial^{i} P(0) \in[-1,1] \times\right.$ $[-2 \epsilon, 2 \epsilon]\}$ and $A^{\prime}$ a neighborhood of $\left\{P \in J_{0}^{2} \mathbb{R}^{2}: P(0) \in[-1 / 2,1 / 2] \times\right.$ $\left.[-1 / 2,1 / 2] \partial^{i} P(0) \in[-1 / 2,1 / 2] \times\left[-\frac{3}{2} \epsilon, \frac{3}{2} \epsilon\right]\right\}$. 
Let us fix an affine $C^{d}$-para-IFS $\left(\left(\stackrel{\circ}{g}_{a}^{\delta}\right)_{a \in \mathbb{R}^{k}}\right)_{\delta \in \Delta}$. Let $\dot{\gamma}: x \in[-1,1] \mapsto$ $\left(x, x^{2}\right)$.

Fundamental property of the parablender If $\infty \geq r>d \geq 1$ and $\left|\Lambda_{\delta}\right|(0)<\left|\lambda_{\delta}\right|^{d}(0)$ for every $\delta \in \Delta$, there exist a $C_{A}^{d, r}$-neighborhood $V_{\gamma}$ of the constant family of functions $(\dot{\gamma})_{a}$ and a $C_{A}^{d, r}$-neighborhood $V_{g}$ of $\left(\left(g_{a}^{\delta}\right)_{a}\right)_{\delta}$ such that for every affine-like parablender $\left(f_{a}\right)_{a}$ with inverse branches $\left(\left(g_{a}^{\delta}\right)_{a}\right)_{\delta} \in V_{g}$, every $\left(\gamma_{a}\right)_{a} \in V_{\gamma}$ has its image $\left(\Gamma_{a}=\gamma_{a}([-1,1])\right)_{a}$ which is $C^{d}$-paratangent at $a=0$ to a local unstable manifold of $\left(f_{a}\right)_{a}$.

We recall that $\left(\Gamma_{a}\right)_{a}$ is $C^{d}$-paratangent to a local unstable manifold $\left(W_{l o c}^{u}\left(\underline{\delta} ; f_{a}\right)\right)_{a}$ if there are $C^{d}$-families of points $\left(C_{a}\right)_{a}$ in $\left(\Gamma_{a}\right)_{a}$ and $\left(Q_{a}\right)_{a}$ in $\left(W_{l o c}^{u}\left(\underline{\delta} ; f_{a}\right)\right)_{a}$ such that:

$$
J_{0}^{d}\left(C_{a}\right)_{a}=J_{0}^{d}\left(Q_{a}\right)_{a} \quad \text { and } \quad J_{0}^{d}\left(T_{C_{a}} \Gamma_{a}\right)_{a}=J_{0}^{d}\left(T_{Q_{a}} W_{l o c}^{u}\left(\underline{\delta} ; f_{a}\right)\right)_{a} .
$$

Remark 5 The fundamental property of parablender cannot be satified in the topology $C_{A}^{d, d}$ since the map $a \mapsto T_{Q_{a}} W_{l o c}^{u}\left(\underline{\delta} ; f_{a}\right)$ is in general not of class $C^{d}$.

To prove the fundamental property, we are going to define a sequence of symbols $\left(\delta_{k}\right)_{k \leq-1}$ and a $C^{d}$-family of points $\left(C_{a}\right)_{a}$ of $\left(\Gamma_{a}\right)_{a}$ satisfying the following property:

$\left(H_{1}\right)$ for every $k \leq-1, J_{0}^{d}\left(G_{a}^{k} \circ C_{a}\right)_{a}$ is in $A$, with $G_{a}^{k}=g_{a}^{\delta_{k}} \circ \cdots \circ g_{a}^{\delta_{-1}}$, $\left(H_{2}\right)$ for every $k \leq-1, J_{0}^{d}\left(D G_{a}^{k} \circ T_{C_{a}} \Gamma_{a}\right)_{a}$ is small.

Proof that $\left(H_{1}\right)-\left(H_{2}\right)$ implies the fundamental property By proceeding as in the proof of Thm B. [1], $\left(H_{1}\right)$ implies the existence of a $C^{d}$-curve of points $\left(Q_{a}\right)_{a}$ in $\left(W_{l o c}^{u}\left(\underline{\delta} ; f_{a}\right)\right)_{a}$ such that:

$$
J_{0}^{d}\left(C_{a}\right)_{a}=J_{0}^{d}\left(Q_{a}\right)_{a} .
$$

Note that $J_{0}^{d}\left(G_{a}^{k} \circ Q_{a}\right)_{a}$ is equal to $J_{0}^{d}\left(G_{a}^{k} \circ C_{a}\right)_{a}$ for every $k$ and so it is in $A$, for every $k \leq-1$.

As $\left(g_{a}\right)_{a}$ is a $C_{A}^{d, d+1}$-perturbation of $\left(\stackrel{\circ}{g}_{a}\right)_{a},\left(W_{l o c}^{u}\left(\underline{\delta} ; f_{a}\right)\right)_{a}$ is $C_{A}^{d, d+1}$-close to a be horizontal, by Prop. 1.6 [2]. The same holds for $\left(W_{l o c}^{u}\left(\sigma^{k}(\underline{\delta}) ; f_{a}\right)\right)_{a}$, with $\sigma^{k}(\underline{\delta})=\left(\delta_{i+k}\right)_{i \leq-1}$. Hence, with $L_{a} \in \mathbb{P R}^{1}$ the line tangent to $W_{l o c}^{u}\left(\underline{\delta} ; f_{a}\right)$ at $Q_{a}$, it holds that $J_{0}^{d}\left(L_{a}\right)_{a}$ is small, and $J_{0}^{d}\left(D_{Q_{a}} G_{a}^{k} \circ L_{a}\right)_{a}$ as well. Consequently, by $\left(H_{2}\right), J_{0}^{d}\left(D G_{a}^{k} \circ T_{C_{a}} \Gamma_{a}\right)_{a}$ is close to $J_{0}^{d}\left(D_{C_{a}} G_{a}^{k} \circ L_{a}\right)_{a}$ for every $k \leq-1$.

Let us notice that the action of $T_{Q_{a}} G_{a}^{k}$ of $D G_{a}^{k}$ on $\mathbb{P R}^{1}$ is exponentially expanding at the neighborhood of $L_{a}$. The same holds for $J_{0}^{d}\left(T_{Q_{a}} G_{a}^{k}\right)_{a}$ : it is exponentially expanding at a ball centered at $J_{0}^{d}\left(L_{a}\right)_{a}$ and which contains $J_{0}^{d}\left(T_{C_{a}} \Gamma_{a}\right)_{a}$. Thus they are equal. 
The proof of $\left(H_{1}\right)-\left(H_{2}\right)$ is done by constructing by induction on $n \leq 0$ a sequence of symbols $\delta_{n-1}, \ldots, \delta_{-1} \in \Delta$ such that there exists a $C^{d}$-curve $\left(C_{a}^{n}\right)_{a} \in\left(\Gamma_{a}\right)_{a}$ satisfying:

(a) $C_{0}^{n}$ is in the interior of the domain of $G_{0}^{n}$.

(b) $G_{a}^{n}\left(C_{a}^{n}\right)$ is the point of $G_{a}^{n}\left(\Gamma_{a}\right)$ with the minimal $y$-coordinate.

(c) $J_{0}^{d}\left(G_{a}^{k}\left(C_{a}^{n}\right)\right)_{a}$ is in $A$ for every $n \leq k \leq 0$ and $J_{0}^{d}\left(G_{a}^{n-1}\left(C_{a}^{n}\right)\right)_{a}$ is in $A^{\prime}$.

We observe that any $C^{d}$-curve $\left(C_{a}\right)_{a}$ in $\left(\Gamma_{a}\right)_{a}$, such that $J_{0}^{d}\left(C_{a}\right)_{a}$ is a cluster value of $\left(J_{0}^{d}\left(C_{a}^{n}\right)_{a}\right)_{n}$ satisfies $\left(H_{1}\right)$ by $(c)$. To see that it satisfies also $\left(H_{2}\right)$, let us bring some materials.

Let $\kappa<1$ and let $\eta>0$ be small and such that:

$$
\begin{aligned}
& 1>\kappa \cdot \exp (\eta), \quad 2 \eta \exp (\eta)+\eta /(1-\kappa \exp (\eta)) \eta<d\left(A^{\prime}, A^{c}\right) \text { and } \\
& 1>\kappa>\max _{\delta \in \Delta} \frac{\Lambda_{\delta}}{\lambda_{\delta}^{d}}(0) \exp (\eta) .
\end{aligned}
$$

Let $p_{y}: \mathbb{R}^{2} \rightarrow \mathbb{R}$ be the $2^{\text {sd }}$ coordinate projection. For any $n \leq-1$, and $\delta_{n}^{\prime}, \ldots, \delta_{-1}^{\prime} \in \Delta$, we define the line field:

$$
\begin{aligned}
& L\left(\delta_{n}^{\prime} \cdots \delta_{-1}^{\prime}, f_{a}\right):=\operatorname{ker} D\left(p_{y} \circ g_{a}^{\delta_{n}^{\prime}} \circ \cdots \circ g_{a}^{\delta_{-1}^{\prime}}\right) \text { and } \\
& L\left(\varnothing, f_{a}\right):=\operatorname{ker} p_{y}=\mathbb{R} \times\{0\} .
\end{aligned}
$$

We will define a complete distance on the space of $C_{A}^{d, d}$-line field families and show the following below:

Lemma 6 For $V_{g}$ sufficiently small, there exists a small neighborhood $V$ of $0 \in$ $\mathbb{R}^{k}$ such that for all $n<0$ and $\delta_{n-1}^{\prime}, \ldots, \delta_{-1}^{\prime} \in \Delta_{d}$, the $C_{A}^{d, d}$-distance between the families $\left(L\left(\delta_{n-1}^{\prime} \cdots \delta_{-1}^{\prime}, f_{a}\right)\right)_{a \in V}$ and $\left(L\left(\delta_{n}^{\prime} \cdots \delta_{-1}^{\prime}, f_{a}\right)\right)_{a \in V}$ (restricted to the intersection of their definition domains) is at most $\eta \kappa^{|n|}\left(\lambda_{\delta_{n}^{\prime}} \cdots \lambda_{\delta_{-1}^{\prime}}\right)$. In particular, $\left(L\left(\delta_{n}^{\prime} \cdots \delta_{-1}^{\prime}, f_{a}\right)\right)_{a \in V}$ is $\eta(1-\kappa)^{-1}-C_{A}^{d, d}$-close to the horizontal line field $\left(L\left(\varnothing, f_{a}\right)\right)_{a \in V}$.

Proof that $(a-b-c)$ implies $\left(H_{2}\right)$ By $(b)$, the curve $\Gamma_{a}$ is tangent to $L\left(\delta_{n} \cdots \delta_{-1}, f_{a}\right)$ at $C_{a}^{n}$, for every $a$ small. Note also that $D G_{a}^{k} \circ T \Gamma_{a}$ is equal to $L\left(\delta_{n} \cdots \delta_{k-1}, f_{a}\right) \circ G_{a}^{k}$ at $C_{a}^{n}$ for every $n \leq k \leq-1$. Thus $J_{0}^{d}\left(D G_{a}^{k} \circ T \Gamma_{a}\right)_{a}$ is equal to $J_{0}^{d}\left(L\left(\delta_{n} \cdots \delta_{k-1}, f_{a}\right) \circ G_{a}^{k}\right)_{a}$ at $J_{0}^{d}\left(C_{a}^{n}\right)_{a}$.

By $(c), J_{0}^{d}\left(G_{a}^{k} \circ C_{a}\right)_{a}$ is in the compact set $A$ for every $k \leq-1$. Also $J_{0}^{d}\left(L\left(\delta_{n} \cdots \delta_{k-1}, f_{a}\right)\right)_{a}$ is $\eta(1-\kappa)^{-1}$-small by Lemma 6 .

Thus, $J_{0}^{d}\left(D G_{a}^{k} \circ T \Gamma_{a}\right)_{a}$ is uniformly dominated by $\eta(1-\kappa)^{-1} \operatorname{diam} A$ at $J_{0}^{d}\left(C_{a}^{n}\right)_{a}$, among $n \leq k \leq-1$. Hence $\left(H_{2}\right)$ holds true at the cluster value $J_{0}^{d}\left(C_{a}\right)_{a}$ of $\left(J_{0}^{d}\left(C_{a}^{n}\right)_{a}\right)_{n}$. 
Proof of the induction hypothesis $(a-b-c)$ Let $n=0$. Let $C_{a}^{0}$ be the point which realizes the $y$-minimum of $\Gamma_{a}$. As it is $C^{d}$-close to 0 for $V_{\gamma}$ small, its $C^{d}$-jet $J_{0}^{d}\left(C_{a}^{0}\right)_{a}$ is in $A$. Hence by $(i)$, there exists a symbol $\delta_{-1}$ such that $G_{a}^{-1} \circ C_{a}^{0}=g_{a}^{\delta_{-1}} \circ C_{a}^{0}$ has its $C^{d}$-jets at $a=0$ in $A^{\prime}$.

Let $n \leq-1$. Let us assume $\delta_{n-1}, \ldots, \delta_{-1} \in \Delta$ constructed such that $\left(C_{a}^{m}\right)_{a}$ satisfies $(a-b-c)$ for every $n \leq m \leq 0$. We put $L_{m a}:=L\left(\delta_{m} \cdots \delta_{-1}, f_{a}\right)$ for every $n-1 \leq m \leq 0$.

For every $n-1<m \leq 0$, we can extend $\left(L_{m a}\right)_{a}$ on $Y_{e}$ such that nearby $a=0$, the line fields $\left(L_{m a}\right)_{a}$ and $\left(L_{n-1 a}\right)_{a}$ are $\eta \sum_{j=m}^{n} \kappa^{|j|} \lambda_{\delta_{j}} \cdots \lambda_{\delta_{-1}} C^{d, d}{ }_{-}$ close by Lemma 6 .

Therefore, there is a unique point $C_{n-1}(a)$ at which $L_{n-1 a}$ and $T \Gamma_{a}$ are equal, this proves $(b)$. Moreover, $J_{0}^{d}\left(C_{a}^{n-1}\right)_{a}$ and $J_{0}^{d}\left(C_{a}^{m}\right)_{a}$ are $\eta \sum_{j=m}^{n} \kappa^{|j|} \lambda_{\delta_{j}}$ $\cdots \lambda_{\delta_{-1}}-C^{d, d}$-close.

We will define the norm involved, and we will prove the following below:

Lemma 7 For $V_{g}$ sufficiently small, for every $\left(\left(g_{a}^{\delta}\right)_{a}\right)_{\delta} \in V_{g}$, and for every $\delta \in \Delta$, the following map is $\exp (\eta) / \lambda_{\delta}(0)$ Lipschitz:

$$
J^{d}\left(g_{a}^{\delta}\right)_{a}: J_{0}^{d}\left(z_{a}\right)_{a} \in A \mapsto J_{0}^{d}\left(g_{a}^{\delta} \circ z_{a}\right)_{a} \in J_{0}^{d} \mathbb{R}^{2} .
$$

Hence, $J_{0}^{d}\left(G_{a}^{m-1}\left(C_{a}^{n-1}\right)\right)_{a}$ and $J_{0}^{d}\left(G_{a}^{m-1}\left(C_{a}^{m}\right)\right)_{a}$ is less than:

$$
\frac{\eta \kappa^{|m|}}{\lambda_{\delta_{m-1}}} \exp (|m-1| \eta)+\eta \sum_{j=m-1}^{n} \kappa^{|j|} \lambda_{\delta_{j}} \cdots \lambda_{\delta_{m-2}} \exp (|m-1| \eta)
$$

By the first inequality of $(\star)$ and since $1>\left|\lambda_{\delta_{m-1}}(0)\right| \geq 1 / 2$, the above sum is at most $2 \eta \exp (\eta)+\eta /(1-\kappa \exp (\eta))$.

By the second inequality of $(\star)$ and the second part of $(c)$ at step $m$, $J_{0}^{d}\left(G_{a}^{m-1}\left(C_{a}^{n-1}\right)\right)_{a}$ is in $A$ for every $n \leq m \leq 0$. This proves the first part of (c) at step $n-1$.

Note that for $m=n$, we obtained that $J_{0}^{d}\left(G_{a}^{n-1}\left(C_{a}^{n-1}\right)\right)_{a}$ is in $A$ and such that $G_{0}^{n-1}\left(C_{0}^{n-1}\right)$ belongs to the domain of any $g_{0}^{\delta}$, hence $(a)$ is satisfied.

Let $\delta_{n-2} \in \Delta$ be such that $J_{0}^{d}\left(g_{a}^{\delta_{n-2}}\right)_{a}$ sends $J_{0}^{d}\left(G_{a}^{n-2}\left(C_{a}^{n-1}\right)\right)_{a}$ into $A^{\prime}$. Note that the second part of $(c)$ is satisfied.

Proof of Lemma 7 We only need to prove this proposition for $\left(\stackrel{\circ}{g}_{a}\right)$ since the Lipschitz constant depends continuously on the $C^{d, d}$-perturbation.

With $J_{0}^{d}\left(z_{a}\right)_{a}=\sum_{j=0}^{d} z_{j} a^{\otimes j}$, and since $\partial_{z}^{j} \stackrel{\circ}{0}_{0}^{\delta}=0$ for every $j \geq 2$, it holds: 


$$
\begin{aligned}
J^{d}\left(\stackrel{\circ}{g}_{a}^{\delta}\left(z_{a}\right)\right)_{a} & =\sum_{n=0}^{d} \sum_{i+k_{1}+\cdots+k_{j}=n} \frac{\left(\partial_{a}^{i} \partial_{z}^{j} \stackrel{\circ}{g}{ }_{0}^{\delta}\right)}{i ! j !} \cdot\left(\otimes_{l} z_{k_{l}}\right) \otimes a^{\otimes n} \\
& =\sum_{n=0}^{d} \sum_{i+k=n} \frac{\left(\partial_{a}^{i} \partial_{z} \stackrel{\circ}{g}_{0}^{\delta}\right)}{i !} \cdot z_{k} \otimes a^{\otimes n}
\end{aligned}
$$

Hence $J^{d}\left(\stackrel{\circ}{g}_{a}^{\delta}\right)_{a}$ is a linear map with upper triangular matrix in the base $\left(z_{n}\right)_{1 \leq n \leq d}$ and with diagonal equal to $\partial_{z} \stackrel{\circ}{g}_{0}^{\delta} \cdot i d$ which is $\left|\lambda_{\delta}^{-1}(0)\right|$-Lipschitz. Hence there exists $\left(c_{i}\right)_{i \leq d}$ with $c_{i}$ small w.r.t. $c_{j}$ whenever $i<j$, such that for the norm $\sum_{j=0}^{d} z_{j} a^{\otimes j} \in J_{0}^{d} \mathbb{R}^{2} \mapsto \sum_{j \leq d} c_{j}\left\|z_{j}\right\|$, the map $J^{d}\left(\stackrel{\circ}{g}_{a}^{\delta}\right)_{a}$ is $\lambda_{\delta}^{-1}(0) \exp (\eta / 2)$-Lipschitz. By taking $V_{g}$ small in function of $\eta$, we get the lemma.

To prove Lemma 6 , let us order the set $\{(i, j): i+j \leq d\}$ by:

$$
\left.(i, j) \succ\left(i^{\prime}, j^{\prime}\right) \text { iff } i>i^{\prime} \text { or } i=i^{\prime} \text { and } j>j^{\prime}\right) .
$$

Proof of Lemma 6. Let $\delta \in \Delta$, and let $\mathcal{L}(\Omega)$ be the space of $C^{d, d}$-families of line fields $\left(L_{a}\right)_{a \in V}$ over a set $\Omega \subset \mathbb{R}^{2}$. We notice that the following map fixes the horizontal line field $H:(a, z) \mapsto \mathbb{R} \times\{0\}$.

$$
\phi:\left(L_{a}\right)_{a} \in \mathcal{L}\left(Y^{\delta}\right) \mapsto\left(\left(D \stackrel{\circ}{g}_{a}^{\delta}\right)^{-1} \circ L_{a} \circ \stackrel{\circ}{g}_{a}^{\delta}\right)_{a} \in \mathcal{L}\left(Y_{\delta}\right) .
$$

In the identification of $\mathbb{P} \mathbb{R}^{1}$ which associates with a line its slope, $H$ is the zero section and the map $\left(D \mathrm{~g}_{a}^{\delta}\right)^{-1}$ is the multiplication by $\Lambda_{\delta}(a) \lambda_{\delta}(a)$. Thus the map $\phi$ is linear:

$$
\phi\left(L_{a}\right)_{a}=\left(\Lambda_{\delta}(a) \lambda_{\delta}(a) \cdot L_{a} \circ \stackrel{\circ}{g}_{a}^{\delta}\right)_{a} .
$$

Note that Lemma 6 is proved if we show that $\phi$ is $\exp (3 \eta / 4) \Lambda_{\delta}(0) \lambda_{\delta}^{1-d}(0)$ contracting for a $C_{A}^{d, d}$-equivalent norm, independent of $\delta$.

Then, there exist continuous functions $\left(C_{i^{\prime}, j^{\prime}}^{i, j}\right)_{\left(i^{\prime}, j^{\prime}\right)<(i, j)}$ such that, for every small $\left(L_{a}\right)_{a} \in \mathcal{L}\left(Y^{\delta}\right)$ :

$$
\begin{aligned}
\partial_{a}^{i} \partial_{z}^{j} \phi\left(L_{a}\right)_{a}= & \Lambda_{\delta}(a) \lambda_{\delta}(a) \cdot \partial_{a}^{i} \partial_{z}^{j}\left(L_{a}\right) \circ \stackrel{\circ}{g}_{a}^{\delta} \cdot\left(\partial_{z} \stackrel{g}{g}_{a}^{\delta}\right)^{i} \\
& +\sum_{\left(i^{\prime}, j^{\prime}\right) \prec(i, j)} C_{i^{\prime}, j^{\prime}}^{i, j}(z, a) \partial_{a}^{i^{\prime}} \partial_{z}^{j^{\prime}}\left(L_{a}\right) \circ \mathrm{g}_{a}^{\delta}
\end{aligned}
$$

Thus the derivatives $\left[\partial_{a}^{i} \partial_{z}^{j}\right]_{i+j \leq d}$ are bounded from above by an upper triangular matrix with diagonal coefficients at most $\left\|\Lambda_{\delta} \lambda_{\delta} \cdot\left(\partial_{z} \stackrel{o}{g}_{a}^{\delta}\right)^{d}\right\|$. For $V$ 
sufficiently small, the latter is at $\operatorname{most} \exp (\eta / 2)\left|\Lambda_{\delta}(0)\right| \cdot\left|\lambda_{\delta}(0)^{d-1}\right|$. Hence, there exists $\left(c_{i, j}\right)_{i+j \leq d}$ with $c_{i, j}$ small w.r.t. $c_{i^{\prime}, j^{\prime}}$ whenever $(i, j) \prec\left(i^{\prime}, j^{\prime}\right)$, such that the map $\phi$ is $\exp (3 \eta / 4)\left|\Lambda_{\delta}(0)\right| \cdot\left|\lambda_{\delta}(0)^{d-1}\right|$-contracting for the norm $\left\|\left(L_{a}\right)_{a}\right\|=\sum_{i+j \leq d} c_{i, j}\left\|\partial_{a}^{i} \partial_{z}^{j} L_{a}\right\|_{C^{0}}$.

\section{References}

1. Berger, P., Crovisier, S., Pujals, E.: Iterated functions systems, blenders, and parablenders. In: Conference of Fractals and Related Fields, pp. 57-70. Springer, (2015)

2. Berger, P.: Generic family with robustly infinitely many sinks. Invent. Math. 205(1), 121-172 (2016)

3. Berger, P.: Emergence and non-typicality of the finiteness of the attractors in many topologies. Proc. Steklov Inst. Math. 297(1), 1-27 (2017)

Publisher's Note Springer Nature remains neutral with regard to jurisdictional claims in published maps and institutional affiliations. 